\title{
A distribuição espacial da indústria do vestuário no Brasil
}

\author{
Rudolph F. A. P. Teixeira ${ }^{1}$ \\ Mario A. Bertella ${ }^{2}$
}

\begin{abstract}
Resumo: Este artigo faz uma análise da distribuição espacial da indústria do vestuário no Brasil para os anos de 1997 e 2006. Para tanto, além do referencial teórico, esse trabalho se utiliza das técnicas de análise exploratória de dados espaciais AEDE. Destarte, foi realizada uma análise univariada para o número de indústrias de vestuário existentes entre os estados brasileiros. Rejeitou-se a hipótese nula de ausência de dependência espacial e a construção dos mapas de clusters indicaram padrões de alta e baixa concentração industrial. Por outro lado, também foi realizada uma análise bivariada, confrontando o número de indústrias de vestuário e massa salarial de regiões vizinhas. O resultado revelou dependência espacial, além de mapas de clusters similares para 1997 e 2006. Assim, dois padrões espaciais emergiram, um de alta e outro de baixa concentração industrial. Conclui-se que as economias de aglomeração são as grandes responsáveis pelos resultados encontrados em ambas as análises.
\end{abstract}

Palavras-chave: Indústria do vestuário; análise exploratória de dados espaciais; concentração industrial.

\section{The spatial distribution of the Brazilian clothing industry}

\begin{abstract}
This paper aims to analyse the spatial distribution of the Brazilian clothing industry in 1997 and 2006. In order to achieve this objective, this study used an exploratory spatial data analysis - ESDA. It was made a univariate analysis of the number of the existing clothing industries among Brazilian states. The null hypotheses of the spatial dependence absence was rejected and the construction of cluster maps showed two patterns: a high industrial concentration and a low one. On the other hand, a bivariate analysis was also conducted to permit a study

1 Mestrando em Economia pela FCLAR/UNESP. E-mail: rudolphgp@hotmail.com.

2 Professor-Doutor do Departamento de Economia da FCLAR/UNESP. Docente do Programa de Pós-

Graduação em Economia da mesma universidade. E-mail: mabertella@yahoo.com.br
\end{abstract}


between the number of clothing industries and the wage income of the neighbor regions. The result revealed spatial dependence besides similar cluster maps in both years. Therefore, two spatial patterns emerged: a high and a low industrial concentration. The final conclusion is that agglomeration economies are the main responsible for the results found in both analyses.

Keywords: Clothing industry; exploratory spatial data analysis; industrial clusters.

JEL: C21, L67

\section{Introdução}

O foco da expansão da indústria, antes atrelado às estruturas internas, isto é, intraindústria, agora se volta para as chamadas economias externas, ou para as relações interindústria. Nesse sentido, a mudança da produção das sedes das grandes empresas, dos países desenvolvidos para as redes de produção global, dispersas em países subdesenvolvidos e em desenvolvimento, configura-se em um verdadeiro "deslocamento do centro dinâmico", onde o antigo padrão unilateral dá lugar a um padrão multilateral ou em forma de teia, com empresas independentes e interconectadas.

Diversos autores passaram a investigar a influência da territorialidade e a formação de clusters espaciais na configuração da estrutura input-output transnacional. O trabalho de Dunning (1994) analisa a existência de vantagens comparativas, e o incremento da participação em alguns mercados, como o grande estímulo das firmas para fazer frente aos custos crescentes em um contexto dinâmico e competitivo. Por sua vez, Stopford (1995), considera que, em um ambiente altamente competitivo, as indústrias devem especializar tanto sua produção, quanto sua localização, com vistas a acumular o maior volume de recursos possíveis para enfrentar a próxima rodada competitiva, isto é, manter sua liderança. Já o artigo de Sturgeon (1997), ao analisar o setor de eletrônica nos EUA, procura discorrer sobre a criação das redes de produção e o aparecimento de clusters industriais, como importante consequência das economias de aglomeração.

Outro estudo de bastante destaque na literatura de economia industrial é o trabalho de Gereffi (1994), que investiga, mormente, a organização espacial, em termos globais, da indústria do vestuário. Para esse autor, esta é uma indústria intensiva em trabalho, com produção descentralizada e estrutura horizontal, onde a produção em massa, a padronização e os baixos salários ditam a estrutura geográfica das firmas. A concentração da produção do vestuário em países pobres e em desenvolvimento, que desfrutam de vantagens comparativas espúrias no fator trabalho (menores remunerações), além de fornecer maior flexibilidade organizacional, seria o grande estímulo dessa indústria. 
No Brasil, a importância da indústria do vestuário passa necessariamente pela expressiva mão de obra por ela empregada. De acordo com a Gazeta Mercantil (1998), cerca de 734 mil pessoas participavam da força de trabalho nesse setor em 1997. Dez anos depois, essa mesma estatística atingia a marca de pouco mais de um milhão de pessoas empregadas, ou seja, um acréscimo nominal de quase 43\% (SINDIVESTUÁRIO 2009).

Segundo a mesma fonte, o Brasil produziu cerca de 5,7 bilhões de peças de vestuário em 2006. Ao passo que esse mesmo valor era da ordem de 4,7 bilhões de peças em 1997 (GAZETA MERCANTIL 1998). Isso configura um incremento aproximado de $21 \%$ na produção durante esse período.

Considerando que o Brasil é um país de proporções continentais, a heterogeneidade da renda, da população, do nível de investimentos, da dispersão industrial, entre outros, é algo inerente à geografia desse país. Estados como os da região Sul e Sudeste possuem um bom nível de desenvolvimento econômico, ao passo que estados das regiões Norte e Nordeste são predominantemente subdesenvolvidos.

Destarte, considerando a hipótese básica de Gereffi (1994) de que a indústria do vestuário tende a se localizar próxima a regiões de baixos salários, este artigo pretende investigar a distribuição espacial dessa indústria entre os estados brasileiros. Para tanto, será utilizado o instrumental da análise exploratória de dados espaciais - AEDE. Caso se comprove empiricamente que estados, com uma elevada concentração de indústrias de vestuário, se localizem próximos a estados com baixa massa salarial, aceita-se a hipótese proposta pelo autor para o Brasil, caso contrário, não.

Nesse aspecto, a próxima seção será responsável por inserir o leitor no contexto metodológico da AEDE, mostrando que os resultados gerados por estatísticas espaciais diferem de formulações tradicionais. Já a seção dois expõe a base de dados utilizada. A seção três, por sua vez, busca revelar os resultados e as descobertas desse estudo espacial, e por fim, a seção seguinte se reporta às considerações finais.

\section{Análise Exploratória de Dados Espaciais}

Conforme destacam Perobelli et al. (2006), a análise exploratória de dados espaciais está baseada em aspectos espaciais da base de dados, tratando diretamente de dependência espacial (i.e. associação espacial) e heterogeneidade espacial. Em suma, a finalidade da AEDE é caracterizar a distribuição espacial, os padrões de associação espacial (clusters espaciais), verificar a ocorrência de diferentes regimes espaciais ou outras formas de instabilidade espacial (não estacionariedade) e identificar observações atípicas (i.e. outliers). 
Para que a AEDE seja implementada de maneira eficiente, é necessária a utilização de variáveis intensivas ou espacialmente densas, já que variáveis absolutas podem induzir a enganos na análise. A utilização do total de observações em números absolutos de um fenômeno não é aconselhável, tendo em vista que estes atributos podem estar correlacionados com variáveis de escala, o que geraria correlações espaciais espúrias. Nesse sentido, a divisão da variável de interesse por algum indicador de intensidade como o número de habitantes ou o tamanho da área de uma região, por exemplo, soluciona esse problema (Anselin 2005).

De acordo com Gonçalves (2005: 411), "métodos convencionais, como regressões múltiplas e inspeção visual de mapas, não são formas mais adequadas de lidar com dados georeferenciados, pois não são confiáveis para detectar agrupamentos e padrões espaciais significativos.”

Assim, a AEDE é o método mais indicado para se obter medidas de autocorrelação espacial global e local, observando a influência dos efeitos espaciais por intermédio de instrumentos quantitativos e não pelo "olho humano" (Anselin 1995; Anselin 1998).

Para se mensurar a autocorrelação espacial global, é utilizado o $I$ de Moran global univariado e também sua versão multivariada ou bivariada. Já para se obter medidas de autocorrelação espacial local, são utilizados os indicadores LISA $^{3}$ representados pelo mapa de clusters em sua versão tanto univariada como multivariada.

\subsection{Autocorrelação Espacial Global Univariada}

Em um estudo de AEDE, a primeira condição a ser satisfeita refere-se ao teste da hipótese nula, em que os dados espaciais são distribuídos aleatoriamente, o que significa que atributos de uma região não dependem dos valores desse atributo em regiões vizinhas (Almeida 2008).

Um coeficiente de autocorrelação descreve um conjunto de dados que está ordenado em uma determinada frequência; assim, um coeficiente de autocorrelação espacial descreve um conjunto de dados que está ordenado em uma sequência espacial. Almeida (2008: 6) ainda destaca que "qualquer coeficiente de autocorrelação espacial pode ser construído pela razão de uma medida de autocovariância e uma medida de variação total dos dados”.

Uma forma de calcular a autocorrelação espacial é por meio do $I$ de Moran. Essa é uma estatística que fornece de maneira formal o grau de associação 
linear entre os vetores de valores observados em um tempo $t\left(Z_{t}\right)$ e a média ponderada dos valores dos seus vizinhos, ou as defasagens espaciais $\left(W Z_{t}\right)$ como são mais conhecidos na literatura. Valores do $I$ de Moran maiores ou menores do que o valor esperado $\mathrm{E}(I)=-1 /(\mathrm{n}-1)$ revelam autocorrelação espacial positiva ou negativa, respectivamente (Almeida et al. 2005; Almeida 2008; Perobelli et al. 2005).

Os valores dessa estatística variam entre -1 e +1 , onde -1 representa um coeficiente de correlação linear perfeitamente negativa e +1 representa um coeficiente de correlação linear perfeitamente positiva (Anselin 1995). Para Perobelli et al. (2006), essa estatística pode ser representada através da seguinte notação matricial:

$$
I_{t}=\left(\frac{n}{S_{0}}\right)\left(\frac{Z_{t}^{\prime} W Z_{t}}{Z_{t}^{\prime} Z_{t}}\right)
$$

na qual $\mathrm{t}=\{1,2 \ldots \mathrm{n}\}$.

Nessa equação, $Z_{t}$ é o vetor de $n$ observações para o ano $t$ na forma de desvio em relação à média. $W$ é a matriz de pesos espaciais, que segundo Haddad e Pimentel (2004: 26), pode ser definida como uma matriz quadrada em que: "cada célula $\mathrm{a}_{\mathrm{ij}}$ indica relação existente entre a região i e j em um sistema de $\mathrm{n}$ regiões. A célula $\mathrm{a}_{\mathrm{ij}}$ é nula no caso das regiões não serem vizinhas, caso contrário o valor passa a ser 1". $S_{0}$ é igual a $\sum \sum w_{i j}$, significando que todos os elementos da matriz de pesos espaciais $W$ devem ser somados.

A matriz de pesos espaciais quando é normalizada na linha, isto é, quando a soma dos elementos da linha é igual a 1, fornece a expressão (1) da seguinte forma:

$$
I_{t}=\left(\frac{Z_{t}^{\prime} W Z_{t}}{Z_{t}^{\prime} Z_{t}}\right)
$$

A literatura registra diversos modos de construir a matriz de pesos espaciais, dentre elas as formulações Rainha (Queen) e Torre (Rook). A matriz Rainha considera vizinhas duas regiões que apresentem fronteiras comuns, além dos nós (vértices) comuns. Já a matriz Torre, por definição, é mais simples, considerando vizinhas apenas as regiões que possuam uma fronteira comum (Haddad \& Pimentel 2004).

Outro critério na definição de matrizes é a distância geográfica. A ideia básica é que duas regiões próximas geograficamente têm maior interação espacial. Nesse aspecto, a matriz de $k$-vizinhos mais próximos, $k=3$, que é utilizada 
nesse trabalho, trata-se de uma matriz binária cuja convenção de vizinhança baseia-se na distância geográfica ${ }^{4}$ (medida em quilômetros ou milhas, por exemplo) (Almeida 2008). Formalmente, conforme (Almeida, Perobelli \& Ferreira 2008), tem-se:

$$
\begin{gathered}
\mathrm{W}_{\mathrm{ij}}(\mathrm{K})=0 \text { se } i=j \\
\mathrm{w}_{\mathrm{ij}}=1 \text { se } \mathrm{d}_{\mathrm{ij}} \leq \mathrm{D}_{\mathrm{i}}(\mathrm{k}) e \mathrm{w}_{\mathrm{ij}}(\mathrm{k})=\mathrm{w}_{\mathrm{ij}}(\mathrm{k}) / \sum \mathrm{w}_{\mathrm{ij}}(\mathrm{k}) \operatorname{para} k=1,2, \ldots, n \\
\mathrm{w}_{\mathrm{ij}}=0 \operatorname{se~} \mathrm{d}_{\mathrm{ij}}>\mathrm{D}_{\mathrm{i}}(\mathrm{k})
\end{gathered}
$$

Onde $d_{i j}$ é a distância, medida pelo grande círculo, entre os centros das regiões $i$ e $j . D_{i}(k)$ denota um valor crítico que define o valor de corte para cada região, isto é, a distância máxima para considerar regiões vizinhas à região $i$, onde regiões acima deste ponto não serão consideradas vizinhas da região em questão. $w_{i j}$ é a matriz de pesos espaciais e $k$ representa o número de vizinhos adotados para cada região geográfica, no caso, $k=3$.

Anselin (1995) considera que o I de Moran, por ser uma medida de associação espacial global, não é capaz de revelar padrões de associação espacial local. Para isso, existem duas ferramentas capazes de detectar esse tipo de padrão, o diagrama de dispersão de Moran e os indicadores locais de associação espacial - LISA.

\subsection{Diagrama de Dispersão de Moran}

Segundo Almeida et al. (2005), o diagrama de dispersão de Moran é uma representação do coeficiente de regressão linear por Mínimos Quadrados Ordinários - MQO, mediante um gráfico de duas variáveis y e $W y$, na qual o coeficiente da inclinação da curva de regressão é dado pela estatística $I$ de Moran. A inclinação da curva é obtida pela regressão de $W y$ contra $y$ e esta inclinação fornece o grau de ajustamento.

O diagrama de dispersão de Moran (figura 1) é dividido em quatro quadrantes. Estes quadrantes correspondem a quatro padrões de associação espacial local entre determinadas regiões e seus respectivos vizinhos (Teixeira et al. 2008). 
FIGURA 1. EXEMPLO DE DIAGRAMA DE DISPERSÃO DE MORAN.

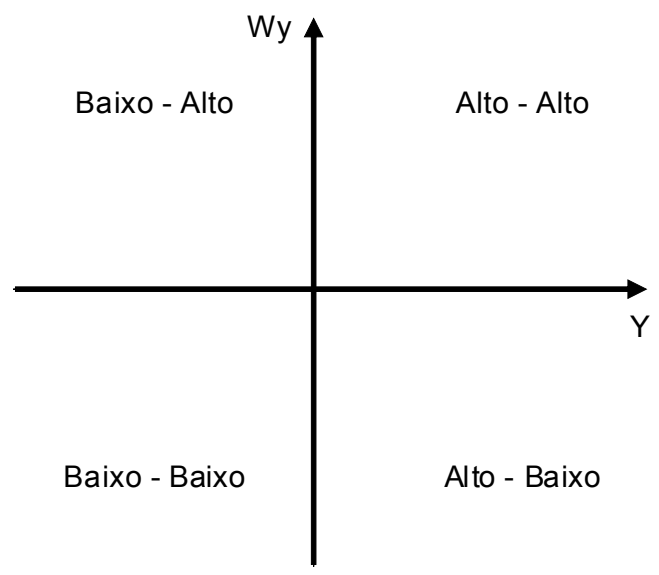

FONTE: Elaboração dos autores.

O primeiro quadrante, conhecido como Alto-Alto (AA), localiza-se na parte superior direita e representa regiões que possuem valores acima da média para uma determinada variável cercada por regiões que também possuam valores acima da média para esta variável.

O segundo quadrante, denominado como Baixo-Alto (BA), localiza-se na parte superior esquerda e é constituído por regiões que apresentam baixos valores para a variável de interesse, rodeadas por regiões que possuem elevado valor para essa mesma variável.

O terceiro quadrante, classificado como Baixo-Baixo (BB), está localizado na parte inferior esquerda e indica regiões que possuem baixos valores para a variável em análise, cercadas por regiões de também baixo valor para a mesma variável.

O quarto e último quadrante, conhecido como Alto-Baixo (AB), encontra-se localizado na parte inferior direita e revela regiões que possuem valores acima da média para a variável de interesse, cercadas por regiões que possuem valores abaixo da média para a mesma variável.

Como destacam Perobelli et al. (2006), as regiões que apresentam padrões de associação espacial positiva AA e BB, formam clusters de valores similares, ao passo que regiões de padrões $\mathrm{BA}$ e $\mathrm{AB}$ apresentam associação espacial negativa. 


\subsection{Indicadores Locais de Associação Espacial (LISA)}

Os indicadores LISA indicam o grau de autocorrelação espacial local. Conforme enfatiza Anselin (1995), para que isso ocorra é necessário que essa estatística satisfaça a dois critérios: a) esses indicadores devem possuir, para cada observação, uma indicação de clusters espaciais significantes de valores similares ao redor de cada observação, e b) o somatório dos indicadores LISA, em todas as regiões, deve ser proporcional ao indicador de autocorrelação espacial global. Dessa maneira, os indicadores LISA podem ser representados por intermédio da seguinte equação:

$$
I_{i, t}=\frac{\left(x_{i, t}-\mu_{t}\right)}{m_{0}} \sum_{j} w_{i, j}\left(x_{i, t}-\mu_{t}\right) \text { em que } m_{0}=\frac{\left(x_{i, t}-\mu_{t}\right)^{2}}{n}
$$

na qual $x_{i, t}$ é a observação de uma variável de interesse na região $i$ para o ano $t, \mu_{t}$ é a média das observações entre as regiões para o ano $t$, no qual o somatório em relação a $j$ é tal que somente os valores vizinhos de $j$ são incluídos.

De acordo com Anselin (1995), a estatística LISA é usada para testar a hipótese nula, ou seja, a ausência de associação espacial local. Assim, deve-se fazer uso de uma aleatorização condicional, que permita determinar pseudoníveis de significância.

Para obtenção de uma distribuição empírica das estatísticas de teste, deve-se observar se o valor da variável de interesse está dentro ou fora da região crítica definida. Dessa maneira, se o valor calculado for superior em magnitude à esperança matemática do $I$ de Moran, seus resultados serão estatisticamente significativos.

Já para se calcular a existência de autocorrelação entre uma dada variável observada em uma região e outra variável observada em uma região vizinha, é requerida a adoção do $I$ de Moran global multivariado. Essa estatística fornece de maneira formal o grau de relacionamento entre as duas variáveis.

\subsection{Autocorrelação Espacial Global Multivariada}

A estatística $I$ de Moran também pode ser utilizada para calcular o grau de interação entre duas variáveis distintas. Conforme enfatiza Almeida (2008), a ideia central é descobrir se valores de uma determinada variável em uma região guardam relação com valores de outra variável em regiões vizinhas.

O coeficiente do $I$ de Moran multivariado ou bivariado com a matriz $W$, já normalizada na linha, é dado pela seguinte equação: 


$$
I^{k, l}=\frac{Z_{k}^{\prime} W Z_{l}}{Z_{k}^{\prime} Z_{l}}
$$

com $n$ representando o número de observações e $W$ a matriz de pesos espaciais. A soma dos quadrados no denominador é constante e igual a $n$ independente

de $Z_{k}$ ou de $Z_{l}$ que representam as duas variáveis distintas padronizadas (Rigotti \& Vasconcellos 2005).

O diagrama de dispersão de Moran multivariado segue o mesmo padrão de análise do diagrama de dispersão de Moran univariado, só que no primeiro o eixo das ordenadas é representado por $W x$, que é a variável $x$ defasada para os vizinhos, confrontada com a variável y no eixo das abscissas (Almeida 2008).

Existem, também, algumas maneiras de se expressar a existência de autocorrelação multivariada em termos locais. Contudo, como forma de manter o estudo homogêneo, será utilizado o $I$ de Moran local multivariado ou bivariado.

\subsection{Autocorrelação Espacial Local Multivariada}

De acordo com Anselin et al., (2003: 7), o I de Moran local multivariado "dá uma indicação do grau de associação linear (positiva ou negativa) entre o valor para uma variável em uma dada locação i e a média de uma outra variável nas locações vizinhas". A estatística $I$ de Moran local multivariado é dada pela equação:

$$
I_{i}^{k, l}=Z_{k i} W Z_{l i}
$$

onde $Z_{k i}$ é a variável padronizada, e $W Z_{l i}$ é a defasagem espacial da variável padronizada. O indicador local de associação espacial (LISA) fornece o grau de autocorrelação espacial, estatisticamente significativo, em cada unidade regional (Haddad \& Pimentel 2004). Destarte, como destaca Almeida (2008), pode-se mapear os valores do $I$ de Moran local bivariado estatisticamente significativos, gerando um mapa de significância bivariado do $I$ de Moran local.

Após explicar a base teórica e metodológica utilizada nesse trabalho, a seguir são detalhados os procedimentos realizados, bem como a base de dados adotada para a elaboração de um estudo espacial da indústria do vestuário nos diversos estados brasileiros. 


\section{Base de Dados}

Este trabalho fez uso do software GEODATA ANALYSIS (GeoDa) e da base de dados da Pesquisa Industrial Anual - PIA, gerada através do SIDRA (Sistema IBGE de Recuperação Automática), do qual se extraíram informações referentes ao número de empresas de vestuário (com mais de 30 funcionários $^{5}$ ) por unidade da federação, além de dados sobre a quantidade total paga de salários, retiradas e outras remunerações (massa salarial), nos distintos estados. Os dados coletados consideram os anos de 1997 e 2006.

A tabela 1 abaixo retrata o número de indústrias e a massa salarial nos anos de 1997 e 2006, bem como suas respectivas taxas de crescimento nos referidos períodos. 
TABELA 1-NÚMERO DE INDÚSTRIAS E MASSA SALARIAL: 1997-2006.

\begin{tabular}{|c|c|c|c|c|c|c|}
\hline \multirow[b]{2}{*}{$\begin{array}{c}\text { Unidades da } \\
\text { Federação }\end{array}$} & \multicolumn{2}{|c|}{ Indústrias } & $\begin{array}{l}\text { Cresci- } \\
\text { mento \% }\end{array}$ & \multicolumn{2}{|c|}{ Massa Salarial } & \multirow{2}{*}{$\begin{array}{c}\text { Cresci- } \\
\text { mento\% }\end{array}$} \\
\hline & 1997 & 2006 & 2006/1997 & $1997^{6}$ & 2006 & \\
\hline Acre & 2 & 6 & 200,0 & 22.520 & 29.825 & 32,4 \\
\hline Alagoas & 28 & 31 & 10,7 & 571.275 & 657.053 & 15,0 \\
\hline Amapá & 2 & 3 & 50,0 & 49.208 & 29.979 & $-39,1$ \\
\hline Amazonas & 16 & 30 & 87,5 & 1.676 .660 & 2.034 .240 & 21,3 \\
\hline Bahia & 292 & 435 & 49,0 & 2.870 .710 & $3 \cdot 377.293$ & 17,6 \\
\hline Ceará & 505 & 1.016 & 101,2 & 1.203 .173 & 1.528 .199 & 27,0 \\
\hline Distrito Federal & 71 & 79 & 11,3 & 384.353 & 380.833 & $-0,9$ \\
\hline Espírito Santo & 317 & 596 & 88,0 & 1.479 .143 & 1.836 .262 & 24,1 \\
\hline Goiás & 573 & 1.182 & 106,3 & 1.114 .823 & 1.775 .484 & 59,3 \\
\hline Maranhão & 36 & 41 & 13,9 & 296.030 & 384.763 & 30,0 \\
\hline Mato Grosso & 33 & 95 & 187,9 & 488.895 & 800.595 & 63,8 \\
\hline $\begin{array}{l}\text { Mato Grosso } \\
\text { do Sul }\end{array}$ & 43 & 106 & 146,5 & 368.768 & 520.766 & 41,2 \\
\hline Minas Gerais & 2.226 & 3.722 & 67,2 & $9.845 \cdot 478$ & 9.927 .505 & 0,8 \\
\hline Pará & 40 & 73 & 82,5 & 911.110 & 1.044 .010 & 14,6 \\
\hline Paraíba & 107 & 130 & 21,5 & 421.905 & 474.760 & 12,5 \\
\hline Paraná & 800 & 2.343 & 192,9 & 5.959 .088 & 7.073 .245 & 18,7 \\
\hline Pernambuco & 278 & 641 & 130,6 & 1.891 .825 & 1.730 .976 & $-8,5$ \\
\hline Piauí & 51 & 132 & 158,8 & 140.030 & 164.865 & 17,7 \\
\hline Rio de Janeiro & 1.636 & 1.838 & 12,3 & 11.317 .603 & 10.480 .317 & $-7,4$ \\
\hline $\begin{array}{l}\text { Rio Grande do } \\
\text { Norte }\end{array}$ & 91 & 187 & 105,5 & 640.645 & 772.006 & 20,5 \\
\hline $\begin{array}{l}\text { Rio Grande do } \\
\text { Sul }\end{array}$ & 858 & 998 & 16,3 & 9.313 .108 & 9.276 .644 & $-0,4$ \\
\hline Rondônia & 13 & 41 & 215,4 & 120.350 & 203.748 & 69,3 \\
\hline Roraima & 5 & 2 & $-60,0$ & 9.898 & 10.258 & 3,6 \\
\hline Santa Catarina & 1.419 & $3 \cdot 306$ & 133,0 & 6.255 .608 & 6.883 .079 & 10,0 \\
\hline São Paulo & $5 \cdot 437$ & 5.838 & 7,4 & 67.290 .118 & 54.419 .192 & $-19,1$ \\
\hline Sergipe & 26 & 75 & 188,5 & 450.218 & 551.448 & 22,5 \\
\hline Tocantins & 6 & 26 & 333,3 & 34.573 & 84.292 & 143,8 \\
\hline
\end{tabular}

FONTE: Elaboração dos autores com base na Pesquisa Industrial Anual - PIA.

6 Os valores de 1997 foram convertidos para valores de 2006, segundo a média do IGP-DI para o referido período. 
Como pode ser observado na tabela 1, todos os estados brasileiros, exceto o estado de Roraima, apresentaram crescimento no número de indústrias de vestuário instaladas em seus territórios no período 1997-2006. Já com relação à massa salarial para o mesmo período, a maioria dos estados apresentou crescimento real da mesma; no entanto, os estados do Amapá, Pernambuco, Rio de Janeiro, Rio Grande do Sul, São Paulo e o Distrito Federal revelaram um comportamento oposto (decréscimo).

Deve-se destacar que a tabela 1 foi construída com valores brutos, ou absolutos, o que caracteriza somente uma análise quantitativa/qualitativa não espacial. Destarte, ao se utilizar o ferramental da AEDE, procura-se incorporar os efeitos locacionais nas variáveis explicitamente, mostrando que o arranjo espacial das mesmas pode apresentar padrões distintos do exposto na tabela 1 acima.

Conforme enfatiza a literatura sobre AEDE, as variáveis devem ser intensivas ou espacialmente densas, isto é, divididas por algum indicador de intensidade, como a população ou a área da região de interesse. Isto porque as variáveis extensivas tendem a estar correlacionadas espuriamente com a área ou a população total da região sob investigação (Anselin 2005). Dessa forma, procura-se verificar a evolução do padrão espacial da indústria brasileira do vestuário durante esse intervalo de dez anos, através da utilização da estatística univariada exposta anteriormente, bem como a existência de clusters industriais, levando-se em conta a massa salarial de regiões vizinhas, através do ferramental da análise bivariada.

\section{Análise e Discussão dos Resultados}

Na elaboração desse estudo, foi utilizada uma matriz de distância geográfica de $k$-vizinhos mais próximos. Adotou-se $k=3$, ou seja, serão considerados vizinhos de uma dada região os três estados mais próximos. Essa matriz foi escolhida porque mostrou os maiores valores do $I$ de Moran, conforme recomenda Almeida (2008). Entretanto, é importante frisar que as demais matrizes de $k$-vizinhos mais próximos ${ }^{7}$, assim como matrizes de contiguidade definidas pelo critério rainha e torre, apresentaram resultados, também, estatisticamente significativos, revelando a robustez da análise. A seguir, serão discutidos os resultados da análise univariada, para a indústria do vestuário, durante os anos de 1997 e 2006. 


\subsection{Análise Univariada: Indústria do Vestuário, 1997-}

2006

Como forma de verificar a existência ou não de clusters para a indústria do vestuário no Brasil, foi realizada uma análise espacial univariada relativa ao número de indústrias (unidades locais) desse segmento nas unidades da federação. O período de análise engloba os anos de 1997 e 2006. Dessa forma, pretendeu-se verificar como a configuração da produção de vestuário se comportou através do tempo e do espaço em um período de dez anos.

A primeira medida adotada foi o cálculo do $I$ de Moran tanto para o ano de 1997, quanto para o ano de 2006. Essa estatística, por elaborar formalmente o grau de autocorrelação espacial de uma unidade com seus vizinhos, revela a existência ou não de um padrão global significativo. Dessa forma, a tabela 2, logo abaixo, fornece os resultados do $I$ de Moran para os dois anos considerados.

TABELA 2. I DE MORAN UNIVARIADO PARA O NÚMERO DE UNIDADES LOCAIS

\begin{tabular}{cc}
\hline Ano & $I$ de Moran \\
\hline 1997 & 0,53 \\
2006 & 0,33 \\
\hline
\end{tabular}

FONTE: Elaboração dos autores com base no software Geoda.

Para o ano de 1997, o I de Moran forneceu o valor de 0,53, ou seja, pode-se rejeitar a hipótese nula ${ }^{8}$ de ausência de autocorrelação espacial, em nível de $5 \%$ de significância. Ou seja, existe uma forte tendência de dependência espacial global, positiva, entre as indústrias de vestuário em uma unidade da federação e as indústrias relativas a esse mesmo segmento, em estados vizinhos.

Já para o ano de 2006, o I de Moran resultou no valor de 0,33, mostrando que, mais uma vez, pode-se rejeitar a hipótese nula ${ }^{9}$ de ausência de dependência espacial global, em nível de 5\% de significância. Dessa forma, esse valor, mesmo sofrendo uma queda de 1997 para 2006, ainda indica uma forte tendência de autocorrelação espacial entre as indústrias de vestuário em um estado e as indústrias de vestuário em estados considerados vizinhos. 
Para que o leitor possa se familiarizar um pouco mais com essas relações, o diagrama de dispersão de Moran, para os anos de 1997 e 2006, é apresentado logo a seguir, na figura 2. Esse diagrama fornece o aspecto espacial das interações, através da regressão por MQO da variável de interesse, contra sua defasagem espacial, isto é, as regiões consideradas como vizinhas.

FIGURA 2 - DIAGRAMAS DE DISPERSÃO DE MORAN PARA A INDÚSTRIA DO VESTUÁRIO: 1997 e 2006, RESPECTIVAMENTE.

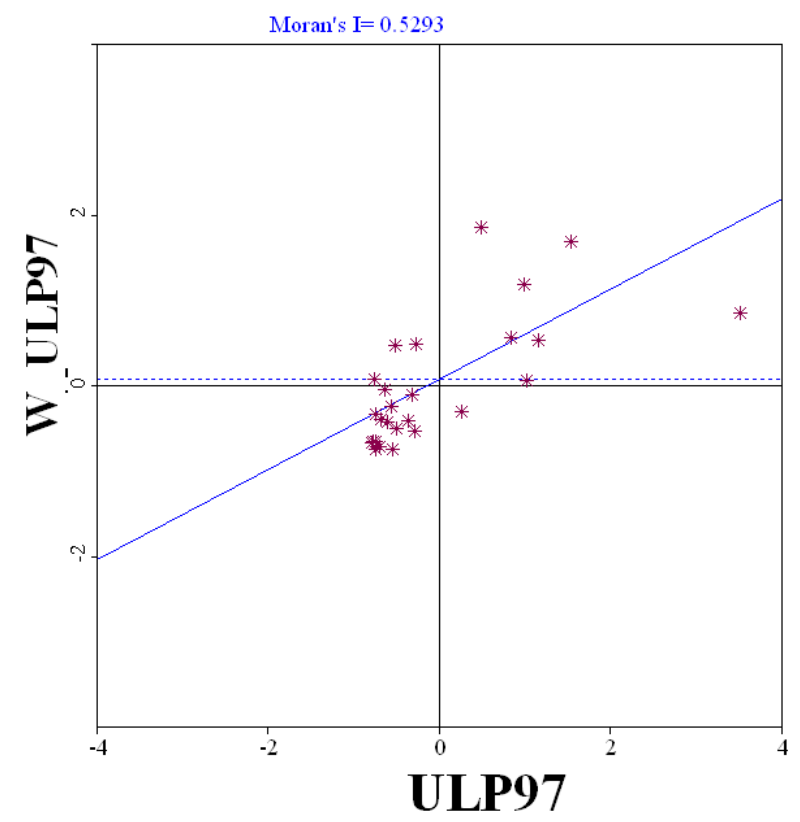




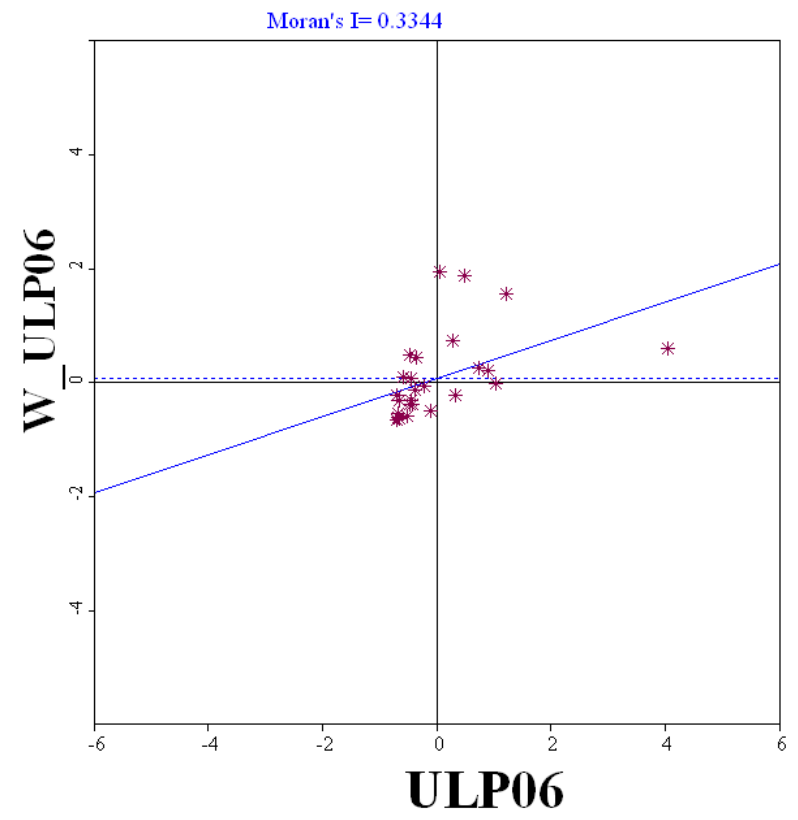

FONTE: Elaboração dos autores com base no software Geoda.

Entretanto, o $I$ de Moran fornece apenas uma estimativa de interação global entre as diversas unidades, considerando-se a variável de interesse. Neste sentido, padrões de interação local tendem a ser omitidos por essa análise, podendo camuflar configurações que destoam da autocorrelação global positiva. A utilização dos indicadores LISA soluciona esse problema, pois através de uma estatística formal, esses indicadores revelam padrões locais de associação espacial, através da geração de mapas. O resultado para a configuração local da indústria do vestuário no Brasil, no ano de 1997, é reportado a seguir pela figura 3 . 


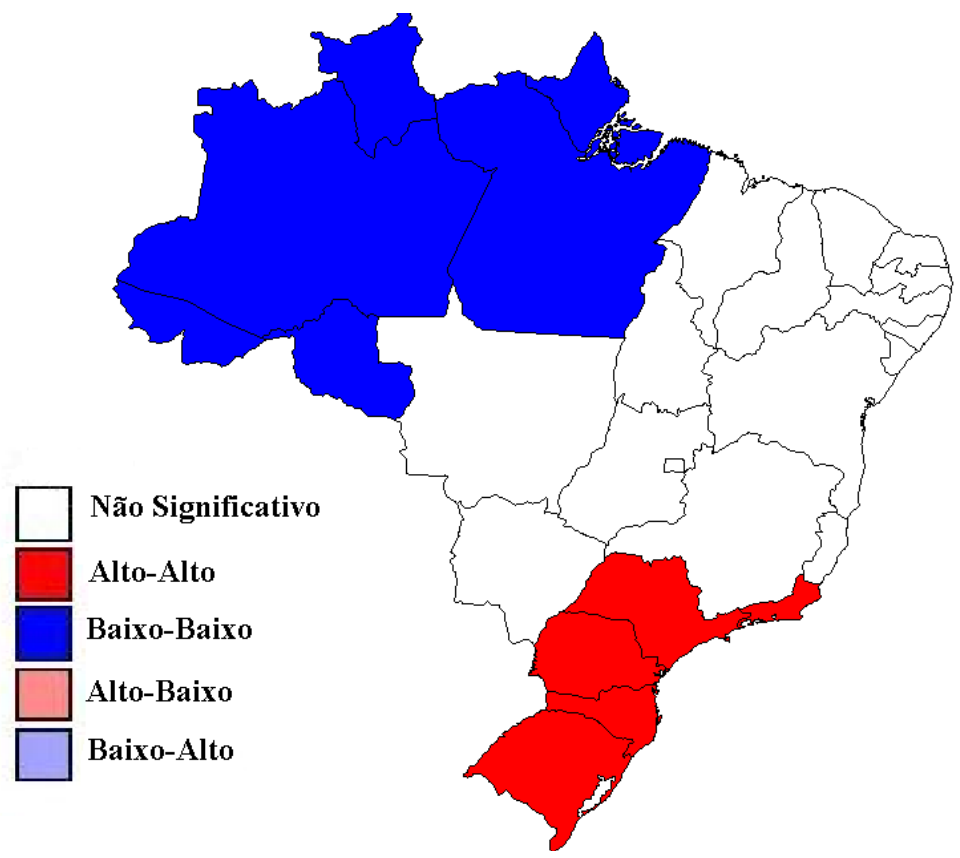

FONTE: Elaboração dos autores com base no software Geoda.

Conforme pode ser visualizado na figura 3, a indústria do vestuário possui dois clusters bem definidos. Um de alta concentração da indústria, o padrão Alto-Alto, e outro de baixa concentração, o padrão Baixo-Baixo. O padrão Alto-Alto indica que regiões, dotadas de um grande número de indústrias do vestuário, são vizinhas de regiões que também possuem um elevado número de indústrias desse segmento. Em 1997, os estados que apresentaram tal configuração foram: São Paulo e Rio de Janeiro, na região sudeste, e todos os estados da região sul, a saber, Paraná, Santa Catarina e Rio Grande do Sul.

Por outro lado, o padrão Baixo-Baixo refere-se a regiões que possuem um número reduzido de indústrias do setor vestuário, que são vizinhas de regiões que, também, detêm poucas indústrias desse segmento. Praticamente, todos os estados da região norte, a saber, Acre, Amazonas, Amapá, Pará, Rondônia e Roraima apresentaram esse padrão, com exceção do estado de Tocantins.

Em relação ao ano de 2006, a configuração do mapa de clusters sofreu poucas alterações, como mostra a figura 4. A existência de dois clusters bem definidos, um de alta concentração industrial e outro de baixa concentração, ainda persiste, como em 1997. Entretanto, o tamanho de ambos se reduziu, isto é, o número de estados com algum desses dois tipos de padrão espacial 
diminuiu.

O padrão Alto-Alto passa a ser composto por todos os estados da região sul do país, ou seja, Rio Grande do Sul, Santa Catarina e Paraná, e pelo estado de São Paulo na região sudeste ${ }^{10}$. Por outro lado, o padrão Baixo-Baixo passa a ser formado por quatro estados da região norte, a saber, Amazonas, Amapá, Pará e Roraima ${ }^{11}$.

\section{FIGURA 4 - MAPA DE CLUSTERS DA INDÚSTRIA DO VESTUÁRIO EM 2006.}

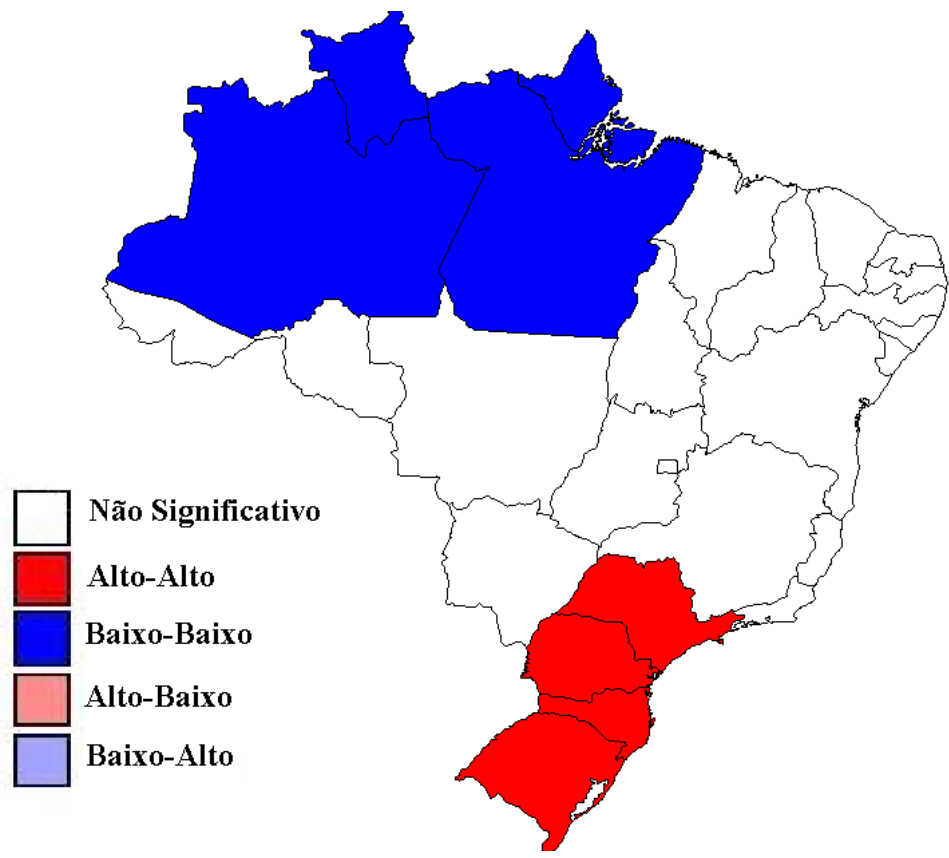

FONTE: Elaboração dos autores com base no software Geoda.

É útil confrontar o mapa de clusters de 1997 com o de 2006 para sugerir tendências de configurações espaciais. Como o padrão Alto-Alto e o padrão Baixo-Baixo reduziram-se de 1997 para 2006, pode-se concluir que um processo de convergência espacial estaria ocorrendo, ou seja, ao longo do tempo, a variável de interesse, isto é, o número de indústrias de vestuário estaria convergindo para um valor comum ao longo das regiões. No entanto, para que tal afirmação seja corroborada, é necessária a utilização de técnicas mais

10 O estado do Rio de Janeiro, presente nesse padrão em 1997, passa a não apresentar mais o padrão AltoAlto, em 2006.

11 Comparando com o ano de 1997, os estados do Acre e Rondônia deixaram de fazer parte desse padrão. 
elaboradas de econometria espacial ${ }^{12}$.

A seguir, como forma de investigar a afirmação de Gereffi (1994) de que regiões que possuem vantagens comparativas espúrias no fator trabalho (que pagam menores salários) tendem a concentrar um elevado número de indústrias do setor vestuário, será realizada uma análise bivariada, comparando a concentração industrial com a massa salarial paga entre os estados brasileiros, por meio de estatísticas formais, como o I de Moran, o diagrama de dispersão de Moran e os indicadores LISA.

\subsection{Análise Bivariada: Indústria do Vestuário e Massa Salarial, 1997-2006}

Para que se possa verificar a existência ou não de autocorrelação espacial entre o número de indústrias de vestuário em uma dada localidade e a massa salarial em regiões vizinhas, calculou-se inicialmente o $I$ de Moran, para os anos de 1997 e 2006. A tabela 3, a seguir, resume os resultados.

TABELA 3 - I DE MORAN BIVARIADO PARA O NÚMERO DE UNIDADES LOCAIS E A MASSA SALARIAL.

\begin{tabular}{cc}
\hline Ano & $I$ de Moran \\
\hline 1997 & 0,61 \\
2006 & 0,57 \\
\hline
\end{tabular}

FONTE: Elaboração dos autores com base no software Geoda.

Considerando inicialmente o ano de 1997, a regressão por MQO da massa salarial contra o número de unidades da indústria do vestuário, forneceu um valor aproximado de 0,61 , ou seja, pode-se rejeitar a hipótese nula ${ }^{13}$ de ausência de dependência espacial, com $5 \%$ de significância. Dessa forma, pode-se inferir que existe uma forte autocorrelação espacial positiva entre essas duas variáveis, em nível global.

Por sua vez, a mesma análise para o ano de 2006 mostra-se extremamente robusta, mesmo com uma ligeira queda do valor do $I$ de Moran para 0,57. Novamente, rejeita-se a hipótese nula ${ }^{14}$ de aleatoriedade espacial, com $5 \%$

\footnotetext{
12 Para saber mais sobre o processo de convergência espacial, veja o trabalho de Rey e Montoury (1999), que aplica a análise da convergência da renda através da abordagem da econometria espacial.

13 O nível de significância para o teste foi de 0,1\% com 999 permutações.

14 O nível de significância para o teste foi de 0,1\% com 999 permutações.
} 
de significância, podendo-se considerar que existe uma forte autocorrelação espacial global positiva entre as indústrias de uma dada localidade e a massa salarial das regiões vizinhas.

Destarte, foi construído o diagrama de dispersão de Moran bivariado (figura 5 abaixo), para os anos de 1997 e 2006, respectivamente, de maneira a auxiliar o leitor na compreensão das interações ditas espaciais, em um contexto bivariado.

FIGURA 5 - DIAGRAMAS DE DISPERSÃO DE MORAN PARA A INDÚSTRIA DO VESTUÁRIO E A MASSA SALARIAL: 1997-2006, RESPECTIVAMENTE.

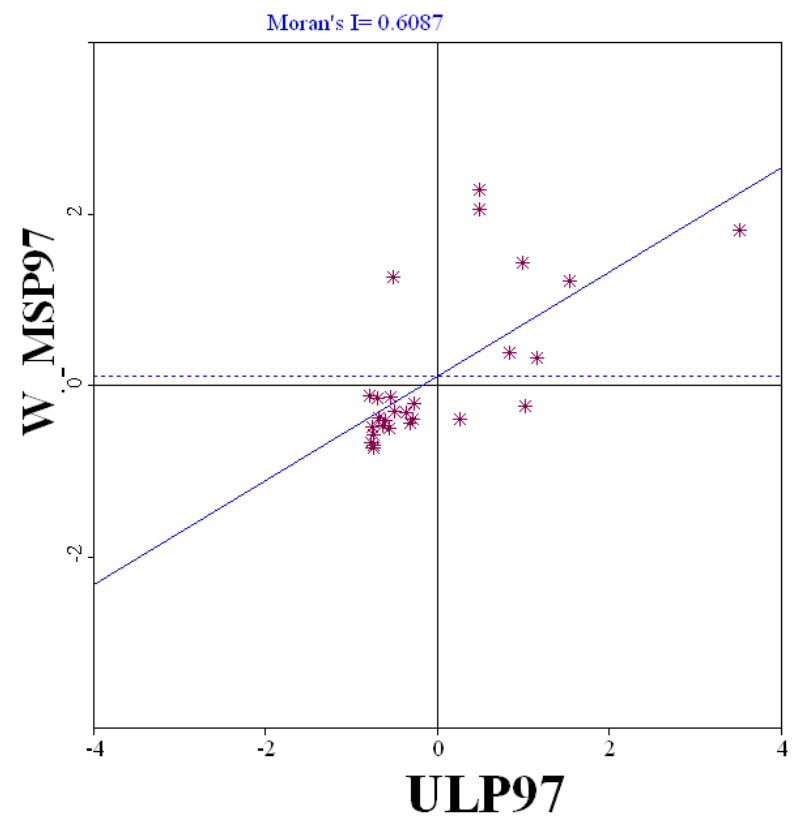




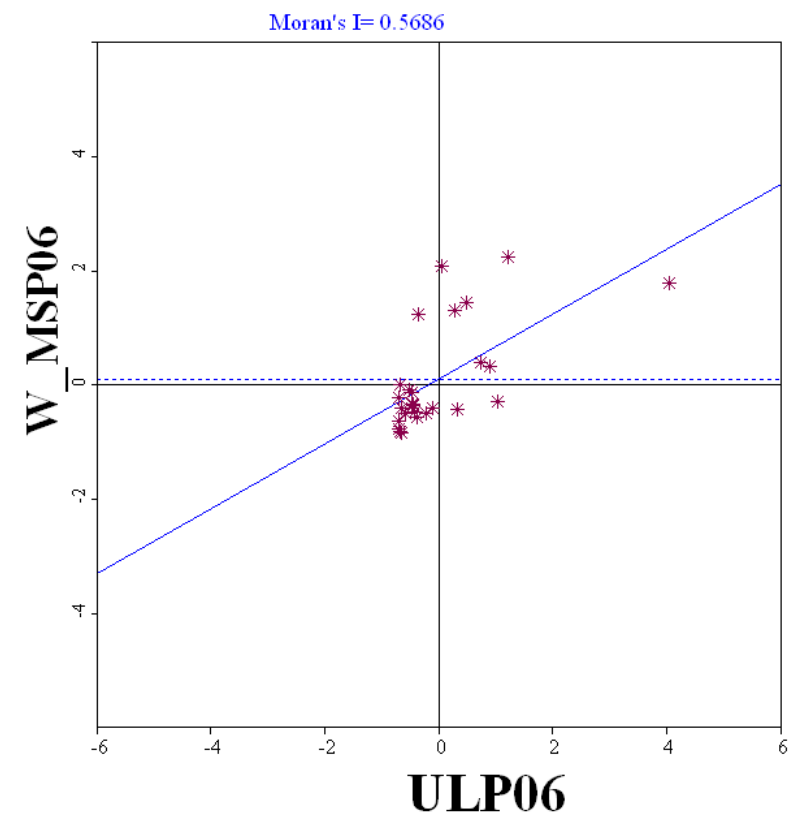

FONTE: Elaboração dos autores com base no software Geoda.

No entanto, como a análise do $I$ de Moran apenas revela a existência ou não de autocorrelação espacial em um contexto global, é importante realizar uma análise de autocorrelação espacial em nível local, e os indicadores LISA cumprem esse papel.

Inicialmente, foi construído o mapa de clusters bivariado para o ano de 1997, confrontando a quantidade de indústrias do vestuário, em um dado estado, com a massa salarial, em estados vizinhos (figura 6). Foram descobertos três tipos de padrões espaciais: o padrão Alto-Alto, o padrão Baixo-Baixo e o padrão Baixo-Alto. 
FIGURA 6 - MAPA DE CLUSTERS BIVARIADO PARA A INDÚSTRIA DO VESTUÁRIO E A MASSA SALARIAL: 1997.

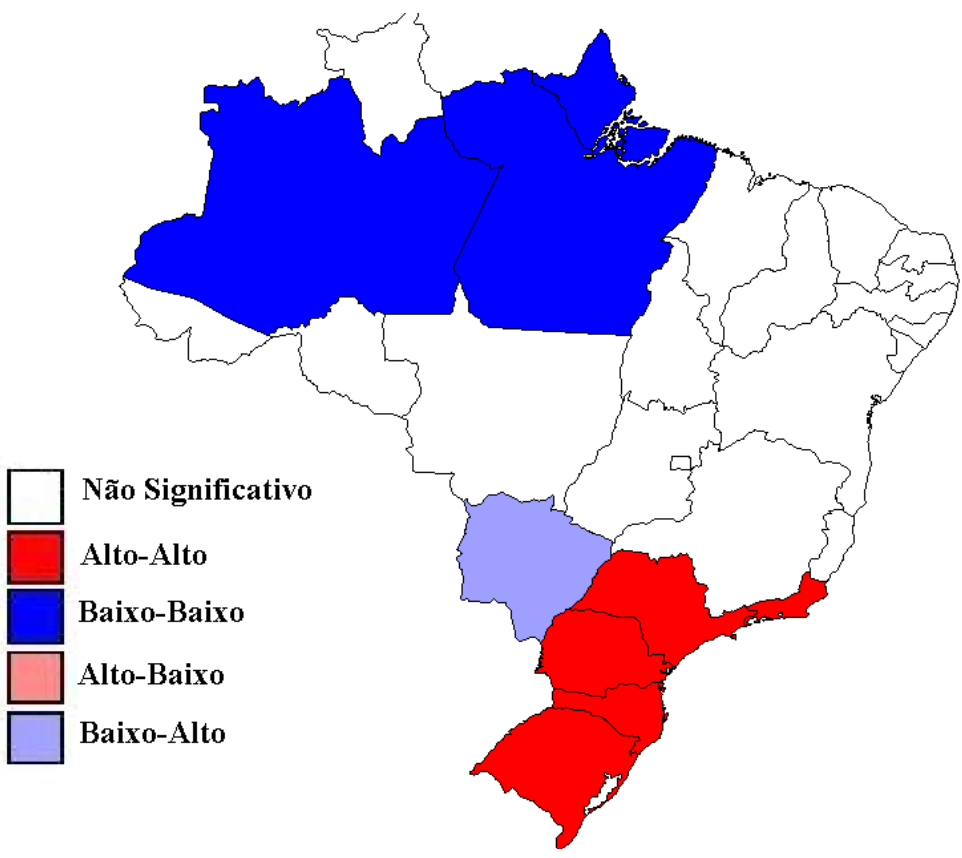

FONTE: Elaboração dos autores com base no software Geoda.

O padrão Alto-Alto indica unidades da federação que possuem uma alta quantidade de indústrias do vestuário, tendo como vizinhas unidades que possuem uma elevada massa salarial. Esse tipo de configuração é encontrado em todos os estados da região sul, Rio Grande do Sul, Santa Catarina e Paraná, além dos estados de São Paulo e Rio de Janeiro, na região sudeste.

Já o padrão Baixo-Baixo revela estados que possuem um reduzido número de indústrias do segmento vestuário circundados por estados que possuem uma baixa massa salarial. Os estados do Amazonas, Pará e Amapá, na região norte, foram os que apresentaram essa configuração.

Por sua vez, o padrão Baixo-Alto apresenta regiões com um limitado número de indústrias do setor de vestuário, cercadas por regiões com uma elevada massa salarial. Esse tipo de configuração foi observado somente no estado de Mato Grosso do Sul, pertencente à região centro-oeste. 
Realizando a mesma análise de mapa de clusters para o ano de 2006, verifica-se que todos os padrões foram mantidos (figura 7). Isto é, novamente foram encontrados os padrões Alto-Alto, composto pelos estados do Rio Grande do Sul, Paraná, Santa Catarina, São Paulo e Rio de Janeiro. O padrão Baixo-Baixo contendo os estados do Amazonas, Pará e Amapá e o padrão Baixo-Alto representado pelo estado do Mato Grosso do Sul.

FIGURA 7 - MAPA DE CLUSTERS BIVARIADO PARA A INDÚSTRIA DO VESTUÁRIO E A MASSA SALARIAL: 2006

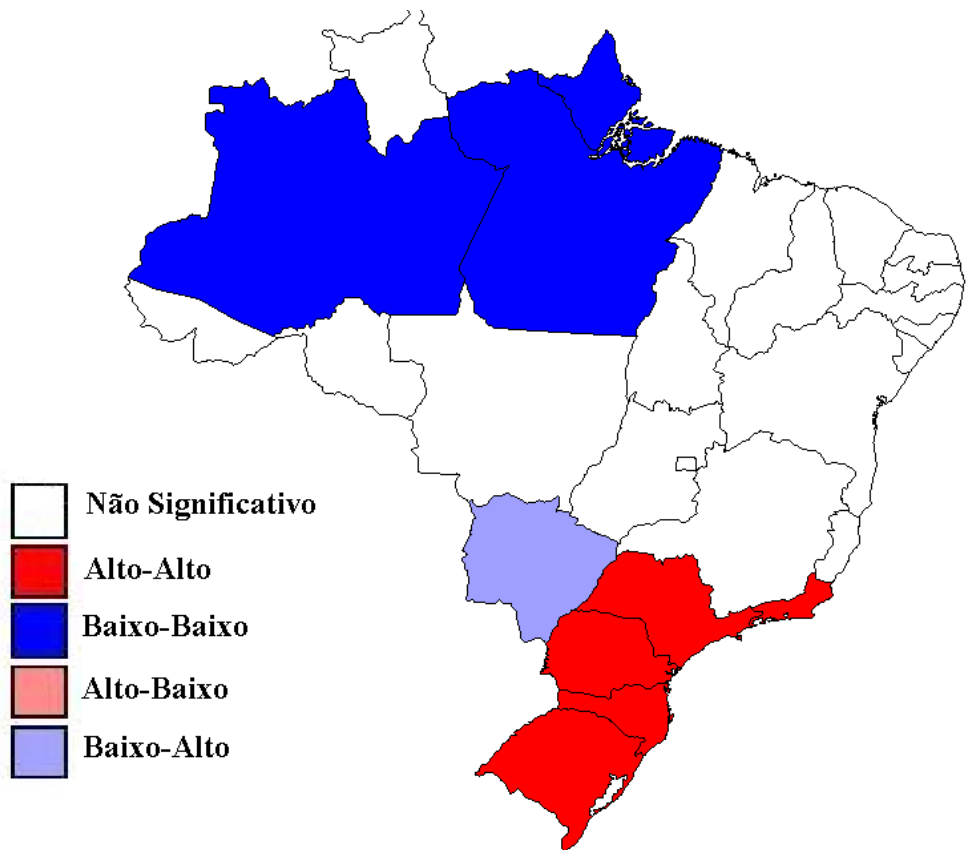

FONTE: Elaboração dos autores com base no software Geoda.

A análise do mapa de clusters pelo contexto bivariado, entre a indústria do vestuário e a massa salarial, considerando-se os distintos estados, revela uma tendência estacionária de 1997 a 2006, mormente pela configuração geográfica das unidades da federação não ter sofrido nenhuma alteração em um razoável espaço de tempo, dez anos.

Dessa forma, pode-se afirmar que existe um delineamento bastante robusto entre os estados para as duas variáveis em questão. A hipótese de Gereffi (1994), de que a indústria do vestuário dirige sua produção para as regiões que desfrutam de menores salários, não se confirma entre os estados brasileiros, durante o período analisado. Ou seja, considera-se que existem 
dois clusters bem definidos, o padrão Alto-Alto e o padrão Baixo-Baixo ${ }^{15}$. Basicamente, estados que possuem um grande número de indústrias voltadas para a produção de vestuário localizam-se próximas a estados com elevada massa salarial. Por outro lado, estados com poucas fábricas de vestuário encontram-se próximas de regiões de baixa massa salarial. O estado do Mato Grosso do Sul é a única exceção, onde este possui uma quantidade reduzida de unidades produtoras de vestuário, sendo vizinha de estados com elevada renda.

Esses resultados corroboram a hipótese básica de Sturgeon (1997), segundo a qual as economias de aglomeração seriam as responsáveis pela formação de clusters espaciais, ou distritos industriais de setores específicos. Forças centrípetas, como uma elevada massa salarial, mostram-se fatores mais que suficientes para explicar grande parte da concentração espacial da indústria do vestuário, ao mesmo tempo em que forças centrífugas, como uma reduzida massa salarial, são capazes de explicar a desconcentração da mesma indústria.

\section{Considerações Finais}

O surgimento de um novo paradigma, no estudo do comportamento industrial, é confirmado pelo número crescente de trabalhos referentes ao contexto das economias externas ou relações "fora da fronteira da indústria”. A produção das firmas multinacionais, antes realizada de forma unilateral, passa a ser elaborada multilateralmente. Nesse sentido, a distribuição espacial e as vantagens comparativas são fatores que passaram a serem investigados por esse novo referencial teórico.

Gereffi (1994) estudou a configuração espacial da indústria do vestuário, em âmbito global, concluindo que a produção desse setor sofreu uma mudança migrando da sede das multinacionais nos países desenvolvidos para os países pobres e em desenvolvimento, tendo como razões principais as vantagens comparativas espúrias (baixos salários) e a flexibilidade organizacional presentes nesses países.

Destarte, como o Brasil é um país de proporções continentais e possui uma indústria significativa no setor de vestuário, este artigo buscou avaliar a hipótese básica de Gereffi (1994) em um contexto nacional. Ou seja, este trabalho buscou investigar como ocorre a distribuição espacial da indústria do vestuário entre os estados brasileiros e se esse setor tende a estar localizado próximo de regiões onde vigoram baixos salários.

15 O padrão Baixo-Alto é considerado um outlier sendo composto apenas por um estado, Mato Grosso do Sul. 
Para que essas hipóteses pudessem ser testadas formalmente, esse trabalho utilizou-se da Análise Exploratória de Dados Espaciais - AEDE. Essa metodologia, através de estatísticas especiais, procura desvendar padrões espaciais de comportamento das variáveis em análise, corroborando ou não a influência do meio e do espaço sobre elas.

A primeira dessas estatísticas a ser considerada é o $I$ de Moran, que indica o grau de autocorrelação espacial global entre a variável de interesse e sua defasagem espacial, em um contexto univariado. Por sua vez, no contexto bivariado, essa mesma estatística relaciona a variável de interesse com o lag espacial de uma outra variável. O I de Moran testa a hipótese nula de aleatoriedade espacial entre as variáveis contra a hipótese alternativa de dependência espacial, considerando interações globais. Entretanto, como essa estatística não consegue detectar padrões locais de associação espacial, é necessário utilizar-se dos indicadores LISA.

Os indicadores LISA, representados pelo diagrama de dispersão de Moran e pelo mapa de clusters, conseguem estabelecer padrões de associação espacial local que o $I$ de Moran não fornece. Isto é, essas estatísticas, por meio de diagramas e mapas, fornecem a configuração da distribuição da variável de interesse no espaço de maneira didática.

Dessa forma, utilizando-se de dados da Pesquisa Industrial Anual PIA, procurou-se investigar como ocorre a distribuição da indústria do vestuário entre os estados brasileiros e se a hipótese de Gereffi (1994) se confirma nessa situação. As variáveis utilizadas foram o número de indústrias de vestuário e a massa salarial presentes nas unidades da federação, durante os anos de 1997 e 2006.

Primeiramente, foi elaborada uma análise univariada para o número de indústrias de vestuário no Brasil (unidades locais), durante os anos de 1997 e 2006. O I de Moran indicou o valor de 0,53 para o ano de 1997 e o,33 para o ano de 2006, ou seja, rejeitou-se a hipótese nula de ausência de dependência espacial para ambos os períodos, em nível de $5 \%$ de significância.

Por sua vez, a construção do mapa de clusters para 1997 revelou dois tipos de configurações, os padrões Alto-Alto e Baixo-Baixo. O padrão Alto-Alto indica que regiões que possuem um elevado número de fábricas de vestuário são vizinhas de regiões que possuem uma grande quantidade de empresas desse segmento. Os estados de São Paulo, Rio de Janeiro, Paraná, Santa Catarina e Rio Grande do Sul foram os que apresentaram tal comportamento. Já o padrão Baixo-Baixo revela que regiões com poucas indústrias de vestuário são circundadas por regiões que também possuem poucas empresas desse setor. Os estados do Acre, Amazonas, Amapá, Pará, Roraima e Rondônia apresentavam essa configuração, em 1997. 
O mapa de clusters, para o ano de 2006, manteve os dois tipos de padrões anteriores, mas estes sofreram redução em seus tamanhos. O padrão AltoAlto passou a ser composto pelos estados de São Paulo, Paraná, Santa Catarina e Rio Grande do Sul (exclusão do Rio de Janeiro). Por sua vez, o padrão Baixo-Baixo passou a ser formado pelos estados do Amazonas, Amapá, Pará e Roraima (exclusão do Acre e Rondônia).

A redução tanto do cluster Alto-Alto quanto do cluster Baixo-Baixo, de 1997 para 2006, sugere a ocorrência de um processo de convergência espacial, i. e., ao longo do tempo, o número de indústrias de vestuário tenderia a colapsar para um valor comum entre as distintas regiões. No entanto, para confirmar essa hipótese, seria necessário um estudo adicional com outras técnicas da econometria espacial.

Também foi realizada uma análise bivariada, confrontando a variável, unidades locais (número de indústrias de vestuário numa dada localidade), no eixo das abscissas, e a variável massa salarial (salários, retiradas e outras remunerações), no eixo das ordenadas. O procedimento inicial foi o cálculo do $I$ de Moran que revelou o valor de o,61, para o ano de 1997, e o valor de 0,57, para o ano de 2006. Assim, rejeitou-se a hipótese nula de aleatoriedade espacial em nível de $5 \%$ de significância, para ambos os períodos.

Nesse sentido, para se verificar arranjos locais, foi construído tanto o mapa de clusters bivariado para o ano de 1997 quanto para o ano de 2006. O mapa de clusters bivariado para o ano de 1997 indicou a presença de três tipos de configurações. Os padrões Alto-Alto, Baixo-Baixo e Baixo-Alto. O padrão Alto-Alto indica que regiões dotadas de um grande número de firmas do setor vestuário são vizinhas de localidades que desfrutam de uma grande massa salarial. Os estados de São Paulo, Rio de Janeiro, Paraná, Santa Catarina e Rio Grande do Sul apresentaram tal comportamento.

O padrão Baixo-Baixo revela que regiões, com poucas indústrias do setor vestuário, são vizinhas de regiões que detêm uma massa salarial reduzida. Os estados do Pará, Amapá e Amazonas detinham tal configuração. O padrão Baixo-Alto, por sua vez, demonstra que localidades com poucas indústrias do setor de vestuário são circundadas por regiões com uma elevada massa salarial. O estado do Mato Grosso do Sul foi o único a apresentar esse padrão.

Já o mapa de clusters construído para o ano de 2006 mostrou-se exatamente igual ao mapa de clusters do ano de 1997. Isto é, todos os padrões foram exatamente mantidos com os mesmos estados. Esses resultados sugerem robustez na configuração dessas variáveis, ao longo do tempo e do espaço. 
Destarte, a hipótese básica de Gereffi (1994) não se verifica entre os estados brasileiros durante o período de investigação, pois os resultados são exatamente o oposto ao esperado. No entanto, a hipótese sugerida por Sturgeon (1997), que enfatiza os aspectos inerentes às economias de aglomeração como fatores responsáveis pela formação de clusters espaciais ou distritos industriais, parece corroborar bem os resultados. Regiões de alta concentração de indústrias de vestuário tendem a estar próximas de regiões que possuem rendimentos elevados, ao passo que o oposto também é verdadeiro.

\section{Referências}

ALMEIDA, Eduardo Simões de. (2008). Econometria Espacial Aplicada. (mimeo), FEA/UFJF.

ALMEIDA, E. S. \& HADDAD, E. A. \& HEWINGS, G. J. D. (2005). "The spatial pattern of crime in Minas Gerais: an exploratory analysis." Economia Aplicada 9 (1).

ANSELIN, L. (1988). Spatial econometrics: methods and models. Kluwer Academic, Boston.

ANSELIN, L. (1995). "Local indicators of spatial association - LISA.” Geographical Analysis 27 (2):93-115.

ANSELIN, L. (1998). "Interactive techniques and exploratory spatial data analysis.” LONGLEY, P. A. \& GOODCHILD, M. F. \& MAGUIRE, D. J. \& WIND, D. W. (eds). Geographical information system: principles, techniques, management and applications. Wiley: New York. pp. 253-365.

ANSELIN, L. (2005). Exploring Spatial Data with GeoDa: a Workbook. University of Illinois, Urbana-Champaign.

ANSELIN, L. \& BERA, A. (1998). "Spatial dependence in linear regression models with an introduction to spatial econometrics." In: Ullah A. e Giles D. E. (eds.) Handbook of applied economic statistics, Marcel Dekker, New York, pp. 237-289.

ANSELIN, L. \& SYABRI, I. \& SMIRNOV, O. (2003). Visualizing multivariate spatial correlation with dynamically linked Windows. Mimeo, University of Illinois.

CLIFF, A. D. \& ORD, J. K. (1981). Spatial processes: models and applications. Pion, London.

DUNNING, J. H. (1988). Explaining International Production. Londres: Unwin Hyman.

GAZETA MERCANTIL. (2009). Vestuário: Setor Bate Recordes de Produção em 97. São Paulo, 1998. Disponível em URL: http://indexet.gazetamercantil. com.br/arquivo/1998/o1/20/115/VESTUARIO:-Setor-bate-recordes-de- 
produção-em-97.html. Acesso em: 16 de Janeiro de 2009.

GEREFFI, G. (1994). "The Organization of Buyer-Driven Global Commodities Chains: How US Retailers Shape Overseas Production Networks." In: Gereffi, G. e Korzeniewicz, M. (eds.) Commodity Chains and Global Capitalism. Londres: Praeger.

GONÇALVES, E. (2005). "Distribuição Espacial da Atividade Inovadora Brasileira: Uma Análise Exploratória”. Texto para discussão CEDEPLAR n ${ }^{\circ}$ 246. Belo Horizonte: UFMG.

HADDAD, E. A.\& PIMENTEL, E. (2004). “Análise da Distribuição Espacial da Renda no Estado de Minas Gerais: Uma Abordagem Setorial”. Texto para discussão, NEREUS. São Paulo.

ODLAND, J. (1988). Spatial autocorrelation. Sage publications, London.

PEROBELLI, Fernando Salgueiro \& ALMEIDA, Eduardo Simões de \& SOUZA, Murilo Corrêa de \& SOUZA, Rafael Morais de. (2005). "Produção de soja na região sul do Brasil (1991-2003): uma análise espacial”. Texto para discussão NUPE $n^{\circ}$ 23. UFJF.

PEROBELLI, F. S.\& FERREIRA, Pedro Guilherme \& FARIA, Weslem Rodrigues. "Análise de Convergência Espacial do PIB per capita em Minas Gerais: 19752003”. In: XI Encontro Regional de Economia, 2006, Fortaleza. Nordeste: Estratégias de Desenvolvimento Regional. Fortaleza: BNB, 2006.

PEROBELLI, F. S. \& ALMEIDA, Eduardo Simões \& ALVIM, Maria Isabel da Silva A\& FERREIRA, Pedro Guilherme. (2007). "Análise Espacial da Produtividade do Setor Agrícola Brasileiro: 1991-2003.” Nova Economia, 17: 65-95.

REY, J. S. \& MONTOURI, B. D. (1999). "US Regional income convergence: a spatial econometric perspective.” Regional Studies 33(2): 143-156.

RIGOTTI, J. I. R \& VASCONCELLOS, I. R. P. (2005). “Uma análise espacial exploratória dos fluxos populacionais brasileiros nos períodos 1986-1991 e 1995-2000." Anais do IV Encontro Nacional Sobre Migrações da Abep. Rio de Janeiro.

SIDRA - Sistema IBGE de Recuperação Automática (2009). Brasília, 2009. URL: http://www.sidra.ibge.gov.br. Acesso em: o9 de janeiro de 2009.

SINDIVESTUÁRIO - Sindicato dos Vestuários. (2009). URL: http://www. sindivest.org.br/content/news/pagina_Item.asp?content_ID=11\#dados. Acesso em URL: 16 de janeiro de 2009.

STOPFORD, J. (1995) “Competing Globally for Resources.” Transnational Corporations, 4: 34-57.

STURGEON, T. J. (1997). "Does Manufacturing Still Matter? The Organization Delinking of Production from Innovation.” BRIE Working Paper, n. 92B. University of California, Berkeley - Berkeley Roundtable on the International Economy. August. 
TEIXEIRA, R. F. A. P. \& ALMEIDA, E. S. \& OLIVEIRA JR., L. B. \& ALVIM, M. I. S. A. (2008). "Análise Espacial da Produtividade de Óleo Vegetal para Produção de Biodiesel na Zona da Mata Mineira.” Gestão.Org, 6(3): 278-299.

TYSZLER, M. (2006) Econometria Espacial: discutindo medidas para a matriz de ponderação espacial. Dissertação de mestrado, Fundação Getúlio Vargas, São Paulo.

Recebido em: 01 de setembro de 2009 Primeira resposta em: 29 de outubro de 2009 Aceite em: 06 de novembro de 2009 